\title{
Entre pouvoir et autorité. Propriété urbaine et production de l'État à Jigjiga, Éthiopie
}

\author{
Emmenegger, Rony
}

\begin{abstract}
Cet article examine la question des restitutions de propriétés urbaines, comprises comme un espace de négociation de l'autorité politique et de production de l'État. À partir d'observations ethnographiques conduites dans la ville de Jigjiga en Éthiopie, il revient sur le rôle des différentes catégories liées à la propriété légale et à la citoyenneté ethnique dans l'accès au foncier et à la propriété en situation de post conflit. L'article offre ainsi une illustration des façons dont les catégories légales se trouvent enchevêtrées dans des ordres normatifs pluriels participant au règlement des cas de restitutions de propriétés et déterminant le (re)façonnage des relations de propriété. Il montre également comment les catégories de propriété légale et de citoyenneté ethnique, bien que codifiées au sein d'un cadre légal commun, sont susceptibles de renfermer des mécanismes antagonistes à la fois d'accès et d'exclusion. Plus généralement, il s'agit de s'interroger sur les ambiguïtés inhérentes aux processus de formation de l'État et sur les dynamiques complexes en œuvre aux marges de celuici.
\end{abstract}

DOI: https://doi.org/10.3917/polaf.132.0115

Posted at the Zurich Open Repository and Archive, University of Zurich ZORA URL: https://doi.org/10.5167/uzh-93469

Journal Article

Published Version

Originally published at:

Emmenegger, Rony (2013). Entre pouvoir et autorité. Propriété urbaine et production de l'État à Jigjiga, Éthiopie. Politique Africaine, 4(132):115-137.

DOI: https://doi.org/10.3917/polaf.132.0115 


\title{
ENTRE POUVOIR ET AUTORITé. PROPRIéTé URBAINE ET PRODUCTION DE L'ÉTAT JIGJIGA, ÉTHIOPIE
}

\author{
Rony Emmenegger et JeanNicolas Bach
}

\section{Editions Karthala I Politique africaine}

\author{
$2013 / 4-N^{\circ} 132$ \\ pages 115137
}

ISSN 0244-7827

Article disponible en ligne l'adresse:

http://www.cairn.info/revue-politique-africaine-2013-4-page-115.htm

Pour citer cet article :

Emmenegger Rony et Bach JeanNicolas, Entre pouvoir et autorité. Propriété urbaine et production de l'État à Jigjiga, Éthiopie,

Politique africaine, 2013/4 №132, p. 115-137. DOI : 10.3917/polaf.132.0115

Distribution ectronique Cairn.info pour Editions Karthala.

Editions Karthala. Tous droits rerv pour tous pays.

La reproduction ou reprentation de cet article, notamment par photocopie, n'est autoris que dans les limites des conditions gales d'utilisation du site ou, le cas hnt, des conditions gales de la licence souscrite par votre ablissement. Toute autre reproduction ou reprentation, en tout ou partie, sous quelque forme et de quelque manie que ce soit, est interdite sauf accord prlable et rit de l'iteur, en dehors des cas prus par la lislation en vigueur en France. II est prisque son stockage dans une base de donns est alement interdit. 


\section{ENTRE POUVOIR ET AUTORITÉ. PROPRIÉTÉ URBAINE ET PRODUCTION DE L'ÉTAT À JIGJIGA, ÉTHIOPIE}

Cet article examine la question des restitutions de propriétés urbaines, comprises comme un espace de négociation de l'autorité politique et de production de l'État. À partir d'observations ethnographiques conduites dans la ville de Jigiiga en Éthiopie, il revient sur le rôle des différentes catégories liées à la propriété légale et à la citoyenneté ethnique dans l'accès au foncier et à la propriété en situation de postconflit. L'article offre ainsi une illustration des façons dont les catégories légales se trouvent enchevêtrées dans des ordres normatifs pluriels participant au règlement des cas de restitutions de propriétés et déterminant le (re)façonnage des relations de propriété. II montre également comment les catégories de propriété légale et de citoyenneté ethnique, bien que codifiées au sein d'un cadre légal commun, sont susceptibles de renfermer des mécanismes antagonistes à la fois d'accès et d'exclusion. Plus généralement, il s'agit de s'interroger sur les ambiguïtés inhérentes aux processus de formation de l'État et sur les dynamiques complexes en œuvre aux marges de celui-ci.

«En revenant, nous avons retrouvé notre identité, notre culture, notre propriété» 1

L'anthropologie politique et la sociologie politique ont démontré que l'État ne peut plus être considéré comme un acteur ou une entité autonome qui serait placé aux antipodes ou en dehors de la société ${ }^{2}$. L'attention s'est ainsi portée sur l'analyse des champs laissant apparaître des pratiques et processus sociaux complexes au sein de relations de pouvoir plus diffuses ${ }^{3}$.

1. Entretien de l'auteur avec un «ancien» (elder) somali, Jigjiga, 24 avril 2012.

2. Je remercie Perscheng Assef, Jean-Nicolas Bach, Tobias Hagmann, Stephan Hochleithner, Éric Komlavi Hahonou, Craig Hatcher, Ilona Imoberdorf, Christian Lund, Ephraim Pörtner et Timothy Raeymaekers pour leurs commentaires qui ont permis d'améliorer l'argument central de cet article, de même que les quatre relecteurs anonymes de Politique africaine.

3. Voir par exemple T. Hagmann et D. Péclard, «Negotiating Statehood: Dynamics of Power and Domination in Africa», Development and Change, vol. 41, n ${ }^{\circ}$ 4, 2010, p. 543; C. Krohn-Hansen et K. G. Nustad (dir.), State Formation: Anthropological Perspectives, Londres, Pluto Press, 2005, p. 12. 


\section{LE DOSSIER}

116 Propriété et citoyenneté dans l'Afrique des villes

Timothy Mitchell ${ }^{4}$ propose ainsi de concevoir et d'analyser l'État comme le produit structurel de tels processus et pratiques - c'est-à-dire comme une structure produite de façon endogène, même si elle se donne à voir comme distincte et hors de la société. Thomas Hansen et Fin Stepputat ${ }^{5}$ ont montré comment l'imaginaire de l'État, en tant que centre d'autorité de la société, naît et se reproduit à travers le recours aux langages symboliques de l'autorité. Cet article porte sur les «zones grises» 6 entre pouvoir et autorité au sein desquelles les significations sont sans cesse façonnées, négociées, et où le pouvoir est «blanchi» pour devenir autorité légitime. Considérant que l'État est mis en œuvre à travers la construction et la déconstruction de l'autorité politique, cet article revient sur les contradictions inhérentes aux processus de formation de l'État et aux dynamiques complexes aux marges de celui-ci ${ }^{7}$.

L'étude des restitutions de propriétés, en tant que domaine de négociation de l'autorité politique, servira à illustrer mon propos. En Afrique comme ailleurs, la question du retour à la propriété a constitué un enjeu majeur des transformations ayant suivi la disparition des régimes socialistes et/ou des situations de post-conflit, largement étudié depuis le début des années $1990^{8}$. Même si le sens donné à la «restitution » peut varier en fonction des contextes dans lesquels le terme est utilisé, celui-ci peut néanmoins être défini dans son acception générale comme «un acte consistant à honorer des revendications antérieures relatives à un droit de propriété à l'encontre de demandes actuelles concurrentes [...] dans l'idée de rendre justice ${ }^{9} »$. Par ailleurs, en reconnaissant une demande d'accès à la propriété, la question des restitutions de propriétés peut en elle-même représenter une source d'autorité politique. Comme Thomas Sikor et Christian Lund l'ont souligné, «le processus de reconnaissance

4. T. Mitchell, «The Limits of the State: Beyond Statist Approaches and Their Critics», The American Political Science Review, vol. 85, n 1, 1991, p. 77-96.

5. T. B. Hansen et F. Stepputat (dir.), States of Imagination: Ethnographic Explorations of the Postcolonial State, Duke University Press, 2001, p. 8.

6. T. Sikor et C. Lund, "Access and Property: A Question of Power and Authority», Development and Change, vol. 40, $\mathrm{n}^{\circ} 1,2009$, p. 19.

7. V. Das et D. Poole (dir.), Anthropology in the Margins of the State, Oxford, James Currey, 2004, p. 9. 8. Voir M. Blacksell et K. M. Born, «Private Property Restitution: The Geographical Consequences of Official Government Policies in Central and Eastern Europe», The Geographical Journal, vol. 168, n², 2002, p. 178-190; P. Maandi, «The Silent Articulation of Private Land Rights in Soviet Estonia: A Geographical Perspective», Geoforum, vol. 40, n³, 2009, p. 454-464; M. Everingham et C. Jannecke, «Land Restitution and Democratic Citizenship in South Africa », Journal of Southern African Studies, vol. 32, n³, 2006, p. 545-562; J. Unruh, «Property Restitution Laws in a Post-War Context: The Case of Mozambique», African Journal of Legal Studies, vol. 1, n 3, 2005, p. 147-165; K. Verdery, "The Elasticity of Land: Problems of Property Restitution in Transylvania», Slavic Review, vol. 53, n 4, 1994, p. 1071-1109.

9. L. M. Fisher et A. J. Jaffe, «Restitution in Transition Countries», Journal of Housing and the Built Environment, vol. 15, 2010, p. 233 et 248. 
de revendications de propriété contribue à ce que l'institution qui statue sur ces revendications se voie simultanément reconnaître son autorité en la matière ${ }^{10}$ ». Précisément, la question des restitutions de propriétés a constitué une formidable arène dans laquelle les institutions les plus diverses ont cherché à réaffirmer leur autorité après la chute des régimes socialistes et/ou en situation de post-conflit ${ }^{11}$.

D'un point de vue empirique, cet article revient sur les procédés par lesquels les restitutions de propriétés ont eu lieu dans la ville éthiopienne de Jigjiga suite à la chute du régime «socialiste» du därg (1974-1991) ${ }^{12}$. Avec le retour des populations somalies originaires de la ville qui avaient fui sous le régime précédent, et dans le nouveau contexte de reconnaissance de la diversité ethnique au sein du système fédéral mise en place par le Front Démocratique Révolutionnaire du Peuple Éthiopien (FDRPE) après 1991, le retour à la propriété devint un enjeu de revendications majeur. Depuis l'incorporation des basses terres somalies au royaume éthiopien à la fin du XIX siècle $^{13}$, Jigjiga fait partie de ces quelques villes où le gouvernement central a réussi à établir un certain contrôle ${ }^{14}$, alors même que l'expansion de l'État est restée embryonnaire dans la plupart des basses terres orientales du royaume ${ }^{15}$. Mais Jigjiga devint un enjeu essentiel pour la représentation des communautés somalies d'Éthiopie en acquérant le statut de capitale du nouvel État fédéré somali au sein de l'Éthiopie fédérale en $1994^{16}$. Cette trajectoire historique fait de Jigjiga un espace dynamique de renégociation entre les autorités de l'État et les citoyens, et un cas d'étude tout à fait pertinent pour l'analyse de la construction, reconstruction ou déconstruction de l'autorité politique.

L'analyse qui suit se fonde sur des données recueillies au cours de recherches menées à Jigjiga durant huit mois entre 2011 et 2013. Les entretiens individuels se mêlent à des discussions de groupes portant sur l'histoire, le foncier et les infrastructures de la ville. Ces sources ont été complétées par quatre semaines

10. T. Sikor et C. Lund, «Access and Property...», art. cit., p. 1; voir aussi l'introduction de C. Lund à ce dossier.

11. Voir T. Sikor, J. Stahl et S. Dorondel, «Negotiating Post-Socialist Property and State: Struggles over Forests in Albania and Romania», Development and Change, vol. 40, n 1, 2009, p. 172.

12. Därg signifie «comité» en amharique.

13. C. R. Barnes, The Ethiopian State and its Somali Periphery: circa 1888-1948, thèse de doctorat, Faculty of History, Trinity College Cambridge, 2000 ; J. Markakis, Ethiopia: The Last Two Frontiers, New York, James Currey, 2011; R. Reid, Frontiers of Violence in North-East Africa: Genealogies of Conflict since 1800, Oxford, Oxford University Press, 2011.

14. S. Devereux, Vulnerable Livelihoods in Somali Region, Ethiopia, Research Report, University of Sussex, 2006, p. 15.

15. T. Hagmann et M. H. Khalif, «State and Politics in Ethiopia's Somali Region since 1991 », Bildhaan, vol. 6, 2006, p. 26.

16. Les communautés ethniques Somali habitent dans différentes régions de la Corne de l'Afrique. On les retrouve en Somalie bien sûr, mais également en Éthiopie, à Djibouti et au Kenya. 
de recherches dans les archives de l'administration municipale et des tribunaux de Jigjiga. Cette base fournira des exemples empiriques relatifs aux processus de restitutions de propriétés en ville et permettra d'illustrer les négociations auprès de l'autorité politique en proposant une synthèse des enjeux, des pratiques et des discours en présence.

La première partie de l'article offre un aperçu des problématiques liées aux restitutions de propriétés et revient plus en détails sur les conséquences des changements institutionnels et politiques en termes de migrations et d'occupation du territoire. La deuxième partie porte sur l'expression de la citoyenneté ethnique et l'ancrage de l'identité politique dans la propriété urbaine en vue d'une éventuelle légitimation des demandes de restitutions. Plutôt que comme un simple cadre légal supposé distinct, la citoyenneté y est entendue comme émanant des pratiques sociales quotidiennes ${ }^{17}$. Dans une troisième partie, l'analyse est recentrée sur le régime légal codifié de propriété et les façons dont les agents gouvernementaux ont interprété ce cadre légal pour réguler les restitutions de propriétés. Il s'agit ici de mettre en lumière la dimension performative de frontières classificatoires ambiguës dans la production de la loi ${ }^{18}$. La quatrième partie revient sur les façons dont les catégories légales de citoyenneté et de propriété ont été mises en jeu à travers les logiques d'accès ou, au contraire, l'échec de l'accès à la propriété ${ }^{19}$. Il s'agit de démontrer que la prise en compte de la dimension matérielle de l'État est incontournable, de même que l'examen des façons dont les documents, les actes et leur proclamation se trouvent entremêlés ${ }^{20}$. L'article montre donc comment l'accès au foncier et à la propriété a été négocié au travers du processus de restitution, et comment cela est lié à la formation du pouvoir et de l'autorité étatique ${ }^{21}$.

\section{RESTITUTIONS DE PROPRIÉtÉS URBAINES À JIGJigA (1991-2012)}

La question des restitutions de propriétés à Jigjiga a fait suite à plusieurs décennies de conflits ethniques ayant conduit à la chute du gouvernement du därg. La guerre de 1977-1978 avait marqué l'émergence de tensions entre

\footnotetext{
17. Voir S. A. Bezabeh, "Citizenship and the Logic of Sovereignty in Djibouti", African Affairs, vol. 110, n 4, 2011, p. 589.

18. D. Hall, P. Hirsch et T. Murray Li, Powers of Exclusion: Land Dilemmas in Southeast Asia, Honolulu, University of Hawaii Press, 2011, p. 12.

19. Voir J.-C. Ribot et N. L. Peluso, «A Theory of Access», Rural Sociology, vol. 68, n², 2003, p. 153;

D. Hall, P. Hirsch et T. Murray Li, Powers of Exclusion..., op. cit..

20. Voir M. S. Hull, Government of Paper: The Materiality of Bureaucracy in Urban Pakistan, Berkeley, Los Angeles, London University of California Press, 2012.

21. Voir T. Sikor, J. Stahl et S. Dorondel, «Negotiating Post-Socialist...», art. cit., p. 172.
} 
les nationalismes éthiopien et somali dont les retombées pour la population multiethnique de Jigjiga furent destructrices ${ }^{22}$. Puis la prise de la ville par les nationalistes somalis en septembre 1977 eut cette fois pour conséquence le déplacement des résidents originaires des hautes terres ${ }^{23}$. Mais cette victoire fut de courte durée et l'armée éthiopienne reprit la ville en mars 1978, poussant à nouveau la majorité des populations somalies au départ. Rares furent celles qui revinrent s'installer à Jigjiga les années qui suivirent, laissant les groupes originaires des hautes terres y devenir majoritaires (Amhara, Oromo, Gurage et Tigray), alors que les populations arabes furent également contraintes de fuir la ville - très peu d'entre elles reviendront s'y établir ${ }^{24}$.

Les déplacés somalis revinrent quant à eux s'installer à Jigjiga suite au déclenchement de la guerre civile en Somalie à la fin des années 1980, phénomène conjugué aux changements politiques initiés en Éthiopie au début des années $1990^{25}$. Ce retour inversa inéluctablement la configuration ethnique de la ville, désormais en faveur d'une nouvelle majorité somalie ${ }^{26}$. Ces événements expliquent la forte croissance de la population de la ville qui, de

\footnotetext{
22. G. Tareke, «The Ethiopia-Somalia War of 1977 Revisited», The International Journal of African Historical Studies, vol. 33, n 3, 2000, p. 635-667. Il en a été ainsi plus particulièrement pour les groupes ethniques originaires des hautes terres (en majorité Amhara, Oromo, Gurage et Tigray), différentes communautés somali locales (Abeskul, Aquisho, Aertirre, Gadabursi, Gerri, Issa, Jeberre, Reer Ali et d'autres sous-clans) de même que des populations d'origine arabe ou yéménite. 23. Voir Jaallesiyaad.com, The Capture of Jigjiga (Jijiga) in the 1977 War Between Ethiopia and Somalia, 1977, <www.youtube.com/watch?v=oY_KPcZi_rA>.

24. De nombreux marchands arabes s'étaient installés à Jigjiga dès la fondation de la ville à la fin du XIXe siècle. Ils avaient joué un rôle déterminant dans le développement de l'économie urbaine. Voir T. Eshete, A History of Jigjiga Town: 1891-74, Department of History, Addis Ababa University, 1988.

25. Le total des Somali d'Éthiopie rapatriés entre 1991 et 1993 est estimé à plus de 600000 (G. Ambroso, Pastoral Society and Transnational Refugees: Population Movements in Somaliland and Eastern Ethiopia 1988-2000, Working Paper, vol. 65, Brussels, UTUNR Agency, 2002, p. 17). Voir également K. Markos, "The Treatment of Somali Refugees in Ethiopia under Ethiopian and International Law », International Journal of Refugee Law, vol. 9, n³ 3, 1997, p. 367-368.

26. En 1984, seulement $24 \%$ de la population urbaine déclarée se définissait comme Somali, alors que les quatre groupes ethniques majoritaires originaires des hautes terres représentaient $62 \%$ de la population: People's Democratic Republic of Ethiopia (PDRE), Population and Housing Census 1984: Analytical Report on Hararge Region, Addis Ababa, 1989, p. 36. Cependant, la part de la population somali atteignait $62 \%$ en 1994, les groupes originaires des hautes terres ne représentant plus que $36 \%$ de la population urbaine: Federal Democratic Republic of Ethiopia (FDRE), The 1994 Population and Housing Census of Ethiopia: Results for Somali Region, Addis Ababa, 1998, p. 73. En 2009, la population Somali était estimée à $78 \%$, et l'ensemble des quatre groupes des hautes terres n'atteignait plus que $20 \%$, selon les résultats de l'enquête menée par le Plan Structurel de la ville de Jigjiga: Jigjiga City Administration, Jigjiga City Structure Plan: Final Report, Jigjiga, January 2012, p. 83. Ces chiffres sont des estimations en raison de l'absence de données pour la ville de Jigjiga dans le recensement de 2007: FDRE, The 2007 Population and Housing Census of Ethiopia: Statistical Report for Somali Region, Addis Ababa, 2007.
} 
24716 habitants en 1984, atteignit les 125896 habitants en $2007^{27}$. Cette forte croissance de la population somalie fut initialement alimentée par un retour massif des anciens habitants de la ville (auxquels s'ajoutaient leurs enfants) et l'arrivée de populations réfugiées originaires de Somalie. Ces mouvements furent progressivement remplacés par d'autres flux constitués de populations somalies originaires d'autres régions éthiopiennes et venant s'établir à Jigjiga en raison du développement important de la ville en tant que centre politique et carrefour marchand des territoires frontaliers somalis. Mais bien souvent, les rapatriés somalis s'installèrent de façon clandestine dans les maisons des membres de leurs familles, de connaissances ou d'anciens voisins, ou s'établirent à proximité sous des huttes somalies traditionnelles.

Le changement de régime au début des années 1990 incita les Somali à réclamer la restitution de leurs maisons - il s'agissait souvent de revendiquer ses droits sur «la maison du père » - occupées avant la guerre de 1977-1978 et redistribuées entretemps à d'autres habitants de la ville par le gouvernement précédent. Avec le changement de régime, de nombreux cas de restitutions de propriétés furent alors négociés de façon privée entre les rapatriés et les occupants des maisons. Pour beaucoup de rapatriés, cet accès fut rendu possible en s'acquittant d'une faible somme d'argent auprès des occupants de la maison - fussent-ils somalis ou originaires des hautes terres - qui permit à ces derniers de disposer des moyens nécessaires à leur réinstallation à Jigjiga ou ailleurs. Durant la même période, certains rapatriés eurent également recours à la violence, soit de leur propre initiative ou avec le soutien de représentants officiels du gouvernement local et/ou des forces de sécurité. Ces restitutions par la violence concernèrent surtout les habitations occupées par des populations originaires des hautes terres plutôt que par des Somali. Notons néanmoins que la violence a également pu représenter un moyen d'action efficace pour les résidents originaires des hautes terres qui, grâce à des relations plus ou moins directes avec le FDRPE et le secteur de la sécurité, sont parvenus à résister à certaines revendications de Somali rapatriés ${ }^{28}$.

S'il n'existe pas de chiffres relatifs au nombre de restitutions des deux dernières décennies, la plupart des personnes avec qui j'ai pu m’entretenir localement ont estimé à plus de la moitié les cas réglés par le biais de négociations privées. Cette tendance évolua au cours des années 1990 vers des procédures légales et administratives, même si le vide laissé par la chute du

27. People's Democratic Republic of Ethiopia (PDRE), Population and Housing Census 1984..., op. cit., p. 36; Federal Democratic Republic of Ethiopia (FDRE), The 1994 Population..., op. cit., p. 73; FDRE, The 2007 Population..., op. cit., p. 9.

28. Ces relations, décisives à l'époque, avec les services de sécurité locaux, régionaux et fédéraux, demeurent significatives aujourd'hui encore. 
därg ne laissait à l'administration locale qu'une capacité limitée dans la prise en charge des demandes de restitution ou l'établissement des droits de propriété. Au niveau du käbälé (sous-district ${ }^{29}$ ) par exemple, les cabinets auparavant en charge de ces dossiers furent dissous et remplacés temporairement par des Comités de Paix et de Stabilité agissant désormais sous la houlette des forces du FDRPE ${ }^{30}$. Mais ces Comités ne s'immiscèrent guère dans les restitutions de propriété, comptant pour cela sur le FDRPE ${ }^{31}$. En 1995, en effet, le gouvernement éthiopien avait fait de la restitution des propriétés une question fédérale dépendant du ressort de l'Agence éthiopienne des Privatisations, mais cette dernière n'eut aucun impact à Jigjiga ${ }^{32}$. Plutôt, suite à la reconfiguration administrative du pays selon une logique ethnique, conduite de façon centralisée depuis Addis-Abeba, c'est bien à l'intérieur du nouveau paysage institutionnel de Jigjiga que le traitement des restitutions fut désormais pris en charge. Au niveau administratif des käbälé, le changement fut lié au licenciement de l'ensemble des anciens fonctionnaires et à leur remplacement par des agents n'ayant pas collaboré, sous quelque forme que ce soit, avec le gouvernement socialiste - principalement des membres des communautés locales somalies. En contraste avec ces bouleversements au niveau des käbälé, le remplacement des fonctionnaires originaires des hautes terres au sein de l'administration municipale fut bien moins brutal, en raison de la faible main-d'œuvre diplômée ou compétente disponible au sein des communautés somalies - une logique que l'on retrouva aux niveaux supérieurs $\mathrm{du}$ district et du tribunal de zone. Cette logique de substitution au sein de l'administration s'accentua néanmoins en 1994 lorsque Jigjiga devint la capitale du nouvel État fédéré régional somali, accueillant désormais le Parlement régional et la Cour suprême régionale ${ }^{33}$.

29. Le käbälé, ou sous-district (sub-district), représente le plus bas des cinq échelons de la structure gouvernementale éthiopienne.

30. Les Comités de Paix et de Stabilité (Peace and Stability Committees) à Jigjiga étaient formés de onze membres (dont le président, le vice-président, trois membres jouant le rôle de tribunal local, un officier de sécurité et trois membres composant l'équipe de sécurité). En outre, un délégué du FDRPE représentait le douzième membre du Comité.

31. Entretien de l'auteur avec un ancien responsable du Comité de Paix et de Stabilité, Jigjiga, 4 août 2013.

32. "Review of Properties Taken in Violation of the Relevant Proclamations ", Proclamation $n^{\circ} 110$, Negarit Gazeta, 54th year, nº 8, 1995. Néanmoins, selon les avocats plaidant à la Cour de Jigjiga, il n'existe aucun cas connu de restitution traité à Jigjiga qui serait remonté à l'Agence à Addis-Abeba.

33. L'administration régionale a connu une autre vague d'ethnicisation après 2004 avec la mise en place du Business Process Re-engineering (BPR), une mesure liée à la réforme de la fonction publique impulsée par le gouvernement d'Addis-Abeba. Dans le cas de la municipalité de Jigjiga, cela se traduisit notamment par le renvoi de fonctionnaires somali aux connaissances limitées en langue somali. 
Ces reconfigurations transformèrent les institutions en charge de la réglementation de la propriété en des lieux hautement stratégiques quant au traitement des demandes de restitution. Plus précisément, l'administration des habitations dont la restitution était réclamée - il n'était pas rare de trouver plusieurs maisons sur une même parcelle - relevait désormais soit d'un des six käbälé gérés par la municipalité de Jigjiga, soit de l'Agence pour le Logement auparavant placée sous l'autorité directe du ministère des Travaux publics et du Logement d'Addis-Abeba. Alors qu'une partie des Somali de retour à Jigjiga parvinrent à récupérer les maisons «de leurs pères» en sollicitant les responsables du gouvernement local au sein de ces institutions, d'autres rencontrèrent davantage de difficultés en raison des réticences de certains fonctionnaires ou des contestations de la part des résidents originaires des hautes terres occupant les maisons. Bien souvent, la stratégie consistant à influencer les représentants du käbälé en s'assurant en amont d'un soutien à un niveau administratif supérieur s'avérait fructueuse. De telles tentatives vis-à-vis de l'Agence de Logement étaient généralement vaines, cette dernière étant soumise à un contrôle du gouvernement central plus étroit que le käbälé. Aucun des cas traité par l'Agence n'a d'ailleurs été réattribué officiellement. Quant à la municipalité de Jigjiga et ses tribunaux, ils ont vu croître les sollicitations relatives à des litiges opposant la plupart du temps des demandes formulées par des Somali à l'égard de résidents originaires des hautes terres - les parties redoublant alors d'efforts pour s'assurer du soutien du fonctionnaire responsable du dossier. Mais dans ces arènes, les cas de restitutions de propriétés étaient souvent considérés sous l'angle réducteur de leur aspect purement juridique et se perdaient ainsi régulièrement dans des procédures administratives et juridiques sans fin.

Bien que le gros des restitutions ait eu lieu au cours des années 1990, certaines procédures légales et administratives de cas non résolus restaient encore ouvertes pendant la décennie suivante. En effet, l'imbrication des cas relatifs aux habitations et parcelles de terrains urbains, associée au manque de preuves juridiques tangibles, rendait la tâche extrêmement difficile pour les représentants du gouvernement, souvent peu disposés à émettre une décision clôturant définitivement un cas de restitution. Dans un tel contexte, on ne peut s'étonner des nombreux témoignages recueillis selon lesquels des fonctionnaires auraient usé à plusieurs occasions de leur pouvoir au profit d'agents privés en échange de diverses rétributions. Des cas de recours à la violence ou à la menace pour certains règlements ont également pu être évoqués ${ }^{34}$.

34. Voir D. Hall, P. Hirsch et T. Murray Li, Powers of Exclusion..., op. cit., p. 19; N. L. Peluso et C. Lund, «New Frontiers of Land Control: Introduction», Journal of Peasant Studies, vol. 38, n 4, 2011, p. 668-676. 
Récemment, le gouvernement régional a finalement décidé de suspendre le traitement des demandes de restitution, comme l'avait fait le gouvernement fédéral quelques années plus tôt ${ }^{35}$.

\section{ETHNICITÉ ET CONSTRUCTION DE LA CITOYENNETÉ}

Cette section revient sur les modalités de la construction sociale de la citoyenneté (celle-ci étant entendue comme un imaginaire spécifique des relations entre les citoyens et les autorités politiques). Il s'agit d'interroger les façons dont cette construction, à l'intérieur de la nouvelle donne dessinée par le fédéralisme ethnique, a pu servir de justificatif aux Somali de Jigjiga dans leurs demandes de restitutions de propriétés ${ }^{36}$.

Depuis la Constitution fédérale de 1995, le FDRPE a fait de l'ethnicité un principe fondamental de l'organisation de l'État, de la représentation et de la citoyenneté. Dans son article 39, ce texte définit les «Droits des Nations, Nationalités et Peuples » et pose les principes au fondement des États régionaux en garantissant le «droit inconditionnel à l'auto-détermination » pour une ethnie sur un territoire donné. Ces «Nations, Nationalités et Peuples» d'Éthiopie sont constitutionnellement définis comme:

«Un groupe de personnes ayant une culture et des traditions similaires ou qui en partagent de nombreux traits communs, une compréhension mutuelle de la langue, la croyance en une identité commune ou de parenté, des traits psychologiques communs et vivant sur un territoire déterminé et essentiellement contigu ${ }^{37}{ }^{\text {». }}$

La reconnaissance d'une citoyenneté ethnique fit évoluer les dispositions de l'État éthiopien à l'égard de sa population somalie. Ce changement d'attitude s'explique également par la reconnaissance des souffrances endurées par le peuple somali sous les régimes précédents.

L'histoire des relations entre l'État éthiopien et le peuple somali est vue par ce dernier comme celle d'une coercition prenant fin avec l'arrivée du FDRPE

35. Period of Limitation for Submission of Restitution Claims and the Repossession of Public Properties Taken Through Unlawful Restitution, Proclamation n ${ }^{\circ} 572$, Negarit Gazeta, 14th year, $\mathrm{n}^{\circ} 24,2008$.

36. Voir J. Eckert, «Introduction: Subjects of Citizenship», Citizenship Studies, vol. 15, n 3-4, 2010, p. 310 ; N. Yuval-Davis, «Belonging and the Politics of Belonging», Patterns of Prejudice, vol. 40, $\mathrm{n}^{\circ} 3$, 2006, p. 205.

37. Traduction non officielle (NdlA). Voir The Constitution of the Federal Democratic Republic of Ethiopia (FDRE) of 1995, Negarit Gazeta, 1st year, n 1, Art. 39§10, texte original: «group of people who have or share a large measure of common culture and similar customs, mutual intelligibility of language, belief in a common or related identities, a common psychological make-up, and who inhabit an identifiable, predominantly contiguous territory». 
au pouvoir. L'origine de cette histoire, selon les récits des habitants de Jigjiga interrogés, daterait de l'expansion de l'État éthiopien dans les basses terres somalies dès la fin du XIX siècle. Rejoignant l'«hypothèse coloniale» décrite par Tobias Hagmann ${ }^{38}$, ces récits insistent sur la nature oppressive d'un État éthiopien soumettant les populations somalies à une répression permanente. Plus précisément, c'est à la lumière d'un grief historique que l'identité somalie est dépeinte comme «cercle fermé positif ${ }^{39}$ » (positive in-group), à la fois juxtaposée et opposée aux «Éthiopiens», entretenant une représentation locale selon laquelle «Éthiopiens» et «Somali» appartiendraient à deux univers sociaux distincts et exclusifs l'un de l'autre ${ }^{40}$. Rompant avec cette perception, le changement de régime en 1991 est dépeint comme un tournant radical modifiant profondément l'attitude de l'État éthiopien envers «ses citoyens somalis ». Nombreux sont les Somali de Jigjiga à avoir exprimé ce changement d'identification politique en se déclarant désormais «Éthiopien-Somali ». Cette approche réconciliait l'histoire tout en maintenant une distinction entre citoyenneté politique et économico-culturelle. Bien que très controversée dans d'autres arènes ${ }^{41}$, la politique régionale du FDRPE et son approche de la citoyenneté dominent ainsi la scène publique de la capitale régionale et ses discours.

À Jigjiga, l'urbanité a cela de spécifique qu'elle adjoint à cette citoyenneté ethnique des critères d'appartenance particuliers tel que l'attachement émotionnel à une communauté et à un «foyer » ${ }^{42}$. D'une part, la citoyenneté ethnique implique la naturalisation de certaines communautés somalies - à l'instar des résidents autochtones originaires de Jigjiga ou des plaines alentours. D'autre part, une communauté si fortement enracinée est marquée par une perspective historique particulière, construite sur l'expérience d'un passé partagé dont font partie l'État éthiopien et les injustices dont les Somali ont souffert. De plus, la citoyenneté ethnique est en elle-même porteuse d'exclusion, comme l'illustrent les membres des communautés somalies décrivant souvent les groupes ethniques originaires des hautes terres comme un «autre» historique, défini par opposition à la communauté somalie profondément enracinée. Les déplacements antérieurs de populations des hautes terres vers les basses terres sont décrits comme une invasion, tandis que leur participation à l'occupation militaire de la région ou de Jigjiga est souvent mise en avant.

38. T. Hagmann, «Beyond Clannishness and Colonialism: Understanding Political Disorder in Ethiopia's Somali Region, 1994-2004 ", Journal of Modern African Studies, vol. 43, n 4, 2005, p. 513. 39. S. Dorman, D. Hammett et P. Nugent (dir.), Making Nations, Creating Strangers: States and Citizenship in Africa, Leiden, Koninklijke Brill NV, 2007, p. 13.

40. Voir T. Hagmann et M. H. Khalif, «State and Politics», art. cit.

41. Ibid., p. 26.

42. N. Yuval-Davis, «Belonging...», art. cit., p. 197. 
Ce processus de définition de l'«autre» passe également par l'agrégation de différents groupes ethniques devenant sans distinctions des «highlanders» (originaires des hautes terres, ou encore habächa en amharique) assimilés étroitement au pouvoir et à l'histoire de l'État éthiopien dont ils symbolisent $l^{\prime}$ héritage ${ }^{43}$. Cette conception émique de l'«autre» néglige la diversité ethnique et les frontières ethniques floues de la citoyenneté urbaine de Jigjiga telle qu'elle émergea dans l'histoire sociale de la ville ${ }^{44}$.

Dès lors, c'est en termes de droits des autochtones et de premiers occupants que les demandes de restitutions de terres et de maisons sont exprimées à Jigjiga. La construction de l'«autre» fournit ainsi une bonne illustration de ce que Sara Dorman et al. appellent «la construction de la nation par la création des étrangers ${ }^{45}$ ». Dans le cas présent, une identité collective somalie a souvent été mobilisée en vue de délégitimer et d'exclure du processus de restitution les résidents originaires des hautes terres. En fonction des circonstances, d'autres collectifs ont également pu être évoqués ou «imaginés » 46 en vue de soutenir une demande de restitution ou les membres d'une communauté autochtone. Ce fut notamment le cas lors de disputes opposant des Somali de retour à Jigjiga à d'autres s'y installant pour la première fois. Les uns et les autres entrèrent en compétition pour l'accès aux maisons de käbälé dans le contexte d'un immobilier sous pression suite à la forte croissance de la population urbaine. Une lettre rédigée et signée par vingt-huit «anciens» («elders») locaux relayait par exemple la plainte des Somali « de Somalie» qui parvenaient à accéder à des maisons de käbälé, posant un problème pour les demandes de restitutions de la part de ceux qui étaient «originaires de la ville $^{47}$ ». Cette lettre fut adressée à divers services gouvernementaux locaux et régionaux dans l'espoir d'obtenir le soutien de fonctionnaires représentant les Éthiopiens-Somali au sein de l'administration. Si ces litiges étaient, certes, de moindre importance que ceux opposant des Somali à des résidents originaires des hautes terres, ils illustrent bien de quelle façon l'imaginaire d'une communauté autochtone induite par les circonstances peut ébranler un autre imaginaire, celui d'une nation somalie unifiée. Le cas des restitutions

43. Voir J. Markakis, Ethiopia..., op. cit., p. 4.

44. Tibebe Eshete a notamment montré comment l'institution sedqo qui émergea dans le contexte urbain régulait et participait à l'harmonisation des relations ethniques entre les occupants de langue amharique et les communautés somali locales. Voir T. Eshete, A History of Jigjiga Town..., op. cit., p. 102-107.

45. S. Dorman, D. Hammett et P. Nugent (dir.), Making Nations..., op. cit., p. 8.

46. B. Anderson, Imagined Communities: Reflections on the Origin and Spread of Nationalism, Londres/ New York, Verso, 1991.

47. Lettre collective de plainte, 18 janvier 1995, trouvée dans les archives de l'administration municipale de Jigjiga. 
de propriétés à Jigjiga dévoile également l'importance des frontières territoriales et la portée de leur évocation dans l'imaginaire spatial des communautés somalies.

\section{PROPRIÉtÉ ET PRODUCTION DE LA LOI}

Cette section revient d'une part sur les façons dont le cadre légal fut interprété dans les cas de restitutions de propriétés et, d'autre part sur les différentes tentatives pour maintenir une cohérence juridique et le respect de la loi.

La reconnaissance d'une citoyenneté ethnique a conduit à une profonde remise en cause des règles régissant les relations de propriété entre les habitants de la ville et les institutions gouvernementales. Mais quelles en sont les raisons? Comme nous avons pu le voir précédemment, l'évocation de l'identité ethnique renvoie au sentiment d'une injustice historique commise envers les «sujets Somali» en Éthiopie - une injustice qui tend à légitimer leurs demandes de restitutions de propriétés. À Jigjiga, ces restitutions concernaient des objets de valeur ancrés dans des relations de propriété, remettant ainsi en cause des pans entiers du droit jusque-là liés à des institutions telles que le käbälé, la municipalité ou l'Agence pour le Logement. Le plus souvent, les maisons disputées étaient administrées par le käbälé ou l'Agence pour le Logement et louées à des habitants de la ville. Les restitutions concernaient également certaines habitations privées, recensées par la municipalité, qui avaient été soit abandonnées soit récemment construites. Néanmoins, la contradiction inhérente au traitement prioritaire des restitutions «à l'encontre de demandes actuelles concurrentes ${ }^{48}$ » apparut comme étant de plus en plus problématique avec la judiciarisation croissante des cas de restitution dans les années 1990.

D'un point de vue juridique, les demandes de restitutions allaient à l'encontre des relations de propriété telles que définies dans les lois du gouvernement «socialiste». À l'instar des autres régions d'Éthiopie, le foncier urbain de Jigjiga fut nationalisé par le därg à la suite de la Proclamation n 47/1975 portant sur la «Nationalisation du Foncier Urbain et des Résidences

48. M. Blacksell et K. M. Born, «Private Property Restitution...», art. cit., p. 188; voir aussi J. Adam, "Post-Conflict Ambon: Forced Migration and the Ethno-Territorial Effects of Customary Tenure", Development and Change, vol. 41, n³, 2010, p. 401-419. 
Secondaires [Extra Houses] ${ }^{49}$ », abolissant la propriété féodale urbaine et mettant fin au régime de propriété impérial ${ }^{50}$. En théorie, le régime socialiste établissait une distinction entre foncier urbain et propriété foncière, chacune de ces deux catégories répondant à des droits spécifiques. Le foncier urbain devint officiellement «propriété du gouvernement» alors que la propriété foncière demeurait partiellement privée. Ainsi, chaque personne et chaque famille pouvait accéder à une "possession» de terre urbaine par le «droit d'user de la terre» (l'État étant propriétaire de cette dernière), mais n'avait le droit «d'être propriétaire» que d'un seul domicile et d'un seul lieu de commerce en contrepartie de prélèvements fiscaux ${ }^{51}$. Toutes les autres maisons furent considérées comme "extra houses», nationalisées et redistribuées sous forme locative aux habitants de Jigjiga.

Après la chute du därg, la Proclamation n 47/1975 demeura le cadre légal à l'intérieur duquel le gouvernement traitait les demandes de restitutions de propriétés. À l'échelle du pays, le gouvernement de transition éthiopien (1991-1995) intégra en effet l'ensemble des droits de propriété hérités du gouvernement socialiste dans le chapitre constitutionnel plus large portant sur les «Droits de Propriété» dans la nouvelle République fédérale. Le foncier urbain et rural resta ainsi propriété de l'État et déclaré bien «commun des Nations, Nationalités et Peuples d'Éthiopie ${ }^{52}$ ». De plus, les citoyens éthiopiens se voyaient reconnaître l'accès à la propriété privée via les «biens immobiliers» en contexte urbain, dont «le droit de céder, de léguer et [...] de perdre sa propriété, transférer son titre, ou demander des compensations en son nom ${ }^{53}$ ». Le gouvernement de transition utilisa ce principe dans le domaine des restitutions de propriétés, notamment par la «Proclamation relative aux propriétés acquises en violation des proclamations pertinentes » de $1995^{54}$,

\footnotetext{
49. Traduction non officielle (Nd1A). Voir Government Ownership of Urban Lands and Extra Houses Proclamation $n^{\circ} 47$, Negarit Gazeta, 34th year, $n^{\circ} 41$, 1975, version originale: «Nationalization of Urban Land and Extra Houses».

50. Ibid., art. 6. Le régime de propriété impérial fut codifié dans le Code civil de l'Empire d'Éthiopie. Voir le Civil Code of the Empire of Ethiopia Proclamation n ${ }^{\circ} 165$, Negarit Gazeta, 19th year, $\mathrm{n}^{\circ} 2,1960$.

51. Government Ownership of Urban Lands and Extra Houses Proclamation $n^{\circ} 47 \ldots$, op. cit., articles 5 et11.

52. Constitution de la République fédérale démocratique d'Éthiopie, art. $40 \S 3$.

53. Traduction non officielle (NdlA). Texte original: «[...] The right to alienate, to bequeath, and [...] to remove his property, transfer his title, or claim compensation for it», The Constitution of the FDRE, articles $40 \S 1$ et 7. Le gouvernement fédéral introduira par la suite d'autres lois relatives au foncier urbain, souvent selon une logique de baux (lease system). Bien que ces lois aient récemment été appliquées en région somalie, elles n'ont eu aucun impact sur le processus de restitutions. Voir Urban Lands Lease Holding Proclamation n 721, Negarit Gazeta, 18th year, n 4, 2011.

54. A Proclamation Relating to Properties Taken in Violation of the Relevant Proclamations, Proclamation $n^{\circ} 110,1995$.
} 
qui ne faisait en réalité qu'entériner la Proclamation n ${ }^{\circ} 47$ de 1975 pour ce qui concerne l'urbain ${ }^{55}$. Ces développements juridiques fixèrent le cadre règlementaire des restitutions de propriétés à Jigjiga, dont les implications furent ainsi résumées par un juriste travaillant pour le tribunal de la ville: «On a besoin d'établir des catégories et de rassembler des preuves valides. En tant que gouvernement, nous nous devons d'identifier et de distinguer les véritables propriétaires ${ }^{56}$ \%.

Cette idée de nécessité à définir des «catégories» renvoie en réalité à la distinction établie entre maisons "nationalisées» et "non nationalisées», selon la base juridique relative aux restitutions de propriétés en vigueur à Jigjiga. L'enjeu de ces catégories apparut particulièrement au cours des procédures judiciaires visant à qualifier les demandes de restitutions. Cette distinction implique que les demandes visant des propriétés nationalisées ne peuvent être considérées comme légitimes dans la mesure où elles ébranleraient les relations de propriété telles que fixées par la loi. En revanche, la catégorie regroupant les maisons «non nationalisées» laisse entendre que certaines maisons ont été acquises à tort par le gouvernement éthiopien, comme ce fut le cas durant la guerre de 1977-1978, ouvrant ainsi l'espace légal dans lequel il devient possible de revendiquer une propriété. Cette distinction catégorielle (entre maisons «nationalisées » et «non nationalisées») apparaissait d'ailleurs dans la lettre des 28 «anciens » mentionnée plus haut, avant même que la Proclamation n $110 / 1995$ ne soit adoptée ${ }^{57}$. Cet exemple révèle bien entendu l'existence de catégories dans les discours publics au-delà des législations formelles, mais surtout l'existence d'une marge de négociation à la fois pour les pouvoirs publics et pour les citoyens.

Dans cette logique, les catégories juridiques ont été interprétées et se sont traduites en pratiques administratives à travers le processus de restitutions. En effet, requérants et fonctionnaires ont été nombreux à souligner, au cours de nos entretiens, l'illégitimité des demandes relatives à une maison «nationalisée » par la Proclamation n 47 de 1975, et la légitimité des cas relatifs aux maisons «abandonnées» au cours de la guerre. Pourtant, un examen plus approfondi de ces catégories nous montre qu'elles ne sont pas exclusives l'une de l'autre: une «extra house» nationalisée en 1975 pouvait par ailleurs avoir été abandonnée pendant la guerre de 1977-1978. Lors de nos entretiens,

55. Ibid., art. 2§3; la Proclamation initiale fut amendée par la Review of Properties Taken in Violation of the Relevant Proclamations (Amendment) Proclamation $\mathrm{n}^{\circ} 193$, Negarit Gazeta, 6th year, $n^{\circ} 21,2000$.

56. Entretien de l'auteur avec un juriste de Jigjiga, Jigjiga, 15 novembre 2012.

57. Lettre collective de plainte, 18 janvier 1995, trouvée dans les archives de l'administration municipale de Jigjiga. 
une distinction revenait également souvent entre «maisons nationalisées » et «maisons de käbälé», ce qui indique que ces dernières furent administrées par le käbälé à partir de la guerre de 1977-1978 et non à partir de la Proclamation n 47 de 1975. Même si toutes les personnes interviewées ne partagent pas cette interprétation historique, notons que cette façon de catégoriser permet de justifier les demandes de restitution de maisons administrées par le käbälé tout en délégitimant les demandes visant les maisons administrées par l'Agence pour le Logement. Ce faisant, les maisons de käbälé devinrent des objets singuliers de dispute entre les résidents originaires des hautes terres et les requérants somalis - disputes qui furent négociées au sein des institutions du gouvernement. Cela montre également comment, dans le contexte spécifique de catégories aux frontières floues, l'enjeu majeur consistait davantage à faire entrer une maison dans une catégorie précise, plutôt qu'à défaire puis reconstruire la trajectoire historique des relations sociales qui fait leur valeur et leur donne sens.

La porosité des frontières entre ces catégories a néanmoins favorisé l'établissement d'un système cohérent pour la régulation des restitutions de propriétés. Cela permit par exemple aux fonctionnaires de reconnaître des demandes ou des contestations de ces dernières via des procédures administratives au niveau du käbälé et de la municipalité, tout en laissant en suspens la contradiction inhérente consistant à prioriser les demandes de restitution «à l'encontre de demandes actuelles concurrentes 58 ». Plusieurs comptes rendus de jugements mentionnent par conséquent les cas de requérants somalis d'abord pris en compte et reconnus par les tribunaux en première instance, mais s'avérant rapidement difficiles à résoudre. En effet, suite aux recours adressés par les résidents originaires des hautes terres, les décisions des tribunaux en première instance furent ultérieurement contredites par les instances supérieures (Cour de district ou Cour suprême), et les demandes de restitution par conséquent rejetées. Ces rejets furent motivés par le fait que les demandes de restitution avaient été reconnues «malgré le fait que [les maisons] avaient été transférée[s] conformément à la Proclamation $n^{\circ} 47 / 1975^{59}$ ». Tout en remettant en cause la «base légale» des premières décisions prises, ces décisions ultérieures invitaient donc à la prudence quant à l'expulsion des résidents, soulignant les risques de «conflits communautaires» pouvant en découler. En résumé, les tribunaux considéraient qu'en ébranlant la structure existante des relations de propriété, la reconnaissance des demandes de restitution représentait une grave menace pour la légitimité du gouvernement.

58. M. Blacksell et K. M. Born, «Private Property Restitution...», art. cit., p. 188.

59. Jigjiga District Administration, lettre enregistrée, 13 novembre 1995. 
La confusion quant à ces restitutions de propriétés suscita une attention accrue de la part du gouvernement après la période de Transition. Le nouveau Parlement de la Région Somali engagea plusieurs initiatives et prit des mesures supplémentaires en vue de se conformer à la loi. Son intervention, notamment en 1996, dans le processus de restitutions des propriétés par l'intermédiaire du Bureau Régional pour l'Administration, la Sécurité, la Justice et la Police (Regional Bureau for Administration, Security, Justice and Police), révèle une vision sécuritaire croissante du processus et confirme l'attention portée au maintien de la paix en ville ${ }^{60}$. Mais son intention était double: d'une part, il s'agissait de renforcer la capacité du gouvernement à évaluer la base juridique applicable aux cas de restitutions et, d'autre part, $d$ 'harmoniser les actions à différents échelons institutionnels. C'est dans ce contexte qu'il faut comprendre la demande émise par le gouvernement régional auprès des institutions gouvernementales subordonnées de lui transmettre l'ensemble des dossiers relatifs aux «maisons possédées par les Somali ayant quitté la ville en $1977^{61}$ ». Au niveau des käbälé et de la municipalité, ce contrôle consista à vérifier jusqu'à la validité des certificats de propriété et des autres documents nécessaires ${ }^{62}$. Mais cette préoccupation croissante visant à se conformer à la loi rendit globalement les modalités de restitutions de propriétés de plus en plus juridiques dans la période qui suivit la Transition.

Dès lors, la mise en place de comités de restitution parut nécessaire étant donné les difficultés à évaluer les bases légales des demandes au sein des diverses institutions du gouvernement ${ }^{63}$. Ces comités furent instaurés à l'initiative du Gouvernement régional somali, particulièrement au niveau de l'administration zonale et de la municipalité, afin de renforcer la capacité des autorités à gérer les demandes de restitution. Les comités de restitution étaient constitués de membres des communautés ayant gagné en importance avec la chute du därg du fait de leur rôle en tant que représentants gouvernementaux au sein de la nouvelle Région Somali ou à leur statut d'« anciens » - une autorité conférée par l'âge ou un rôle de meneur au sein de la structure clanique. Chargés d'examiner les demandes de restitutions, ces comités auditionnaient également des témoins et considéraient les preuves écrites afin de déterminer quelles maisons avaient été - ou non - nationalisées dans le cadre de la Proclamation de 1975. Ces comités auraient ainsi examiné quantité de cas, mais

60. Somali Regional Parliament, lettre enregistrée, 12 décembre 1996.

61. Ibid.

62. Jigjiga City Municipality, lettre enregistrée, 1er janvier 1997, trouvée dans les archives de l'administration municipale de Jigjiga.

63. Lettre collective de plainte, 18 janvier 1995; Jigjiga City Municipality, lettre enregistrée, 13 avril 1999. 
faute de capacité de décision, ils furent finalement incapables de statuer de façon indépendante.

\section{DOCUMENTS ET AUTORITÉS EN NÉGOCIATION}

Cette dernière partie analyse les façons dont la citoyenneté légale et les catégories de propriété ont été manipulées au cours des processus d'accession-ou de non accession - au foncier depuis le début des années 1990. Il s'agit d'illustrer les façons dont cette multiplicité de normes s'est reproduite en pratique et de montrer comment ce processus contribue à faire et défaire l'autorité politique.

À Jigjiga, différents acteurs au sein de l'appareil bureaucratique ou en dehors de celui-ci ont négocié le sens donné aux catégories juridiques de la citoyenneté et de la propriété. Ces négociations ont produit une pluralité d'ordres normatifs dessinant le cadre règlementaire des restitutions de propriétés. Autrement dit, plusieurs catégories juridiques cohabitent désormais à l'intérieur du même corpus législatif et produisent des ordres normatifs qui, en pratique, peuvent servir à justifier les demandes et déterminent la régulation de la propriété ${ }^{64}$. Ce pluralisme a permis aux requérants de recourir au répertoire de la citoyenneté ethnique ou à celui du droit à la propriété pour faire entendre leurs revendications auprès d'une autorité puissante et décisive. Mais il a également offert une plus grande liberté d'interprétation aux fonctionnaires qui peuvent louvoyer d'un ordre normatif à l'autre selon qu'ils souhaitent rendre légitimes ou illégitimes les revendications exprimées.

À Jigjiga, la citoyenneté ethnique s'est inscrite au cœur de puissants discours grâce auxquels l'identité somalie est devenue un outil légitimant dans le cadre des demandes de restitutions. Elle s'est ainsi constituée en «norme pratique ${ }^{65}$ » qui a structuré et régulé les pratiques des fonctionnaires. Notons qu'une telle norme doit être distinguée d'une norme «non officielle», même si elle représente bel et bien une structure située, un produit de la négociation de la règle «officielle» et des catégories légales de propriété. Cette norme pratique a ainsi fourni au maire de Jigjiga, au début des années 2000, le socle de justification à sa décision de chasser des résidents d'une propriété afin de répondre favorablement à la demande de restitution d'une femme âgée.

64. Voir également F. von Benda-Beckmann, "Who's Afraid of Legal Pluralism», Journal of Legal Pluralism, vol. 47, 2002, p. 63; G. R. Woodman, «Ideological Combat and Social Observation: Recent Debate About Legal Pluralism », Journal of Legal Pluralism, vol. 42, 1998, p. 21-59.

65. J.-P. Olivier de Sardan, Researching the Practical Norms of Real Governance in Africa, Discussion paper, $n^{\circ}$ 5, Londres, Africa Power and Politics Programme, 2008. 
Au cours d'un entretien mené en 2012, ce maire somali justifiait son intervention en arguant que cette femme «somalie» avait initialement été forcée de quitter sa maison par les «occupants des hautes terres ${ }^{66}$ ». Il n'est pas rare de trouver des cas similaires où les fonctionnaires ont pu accéder aux requêtes et imposer leur décision en déclarant rendre «justice». La citoyenneté ethnique a donc eu un impact significatif sur les relations ethniques, traversant les identités claniques des Somali et ignorant les structures administratives ou les frontières étatiques. Les requérants ont ainsi eu recours à la citoyenneté ethnique au niveau du käbälé ou de la municipalité pour justifier leur demande de restitution et exclure l'«autre venu des hautes terres», ou les Somali «de Somalie» selon les circonstances. De plus, la citoyenneté ethnique s'est avérée très utile pour justifier l'accès à des terres ou à des habitations dont la propriété avait été acquise auparavant par la violence ou des moyens financiers; elle est devenue aussi un moyen de légitimer l'accès à la propriété au sein du nouvel ordre fédéral ethnique. Et c'est un outil qui s'est également révélé des plus efficaces lorsqu'il s'est agi de rejeter les prétentions du käbälé et de l'Agence pour le Logement à récupérer les maisons réappropriées. Il n'est dès lors pas très surprenant que le directeur de cette Agence ait admis que son institution n’avait ni les moyens ni l'ambition de prélever le loyer mensuel d'individus dont la maison «appartenait à leur père ${ }^{67}$ ».

La judiciarisation croissante du règlement des restitutions après la période de Transition a toutefois rendu plus difficile pour les plaignants d'obtenir un écho favorable auprès des institutions en fondant leur demande de restitution uniquement sur la citoyenneté ethnique. Le traitement d'une telle demande par la municipalité ou le tribunal allait désormais être conditionné par la capacité à présenter des documents légaux appropriés. Le poids des preuves formelles devint par ailleurs bien plus important lorsqu'il s'agissait de délégitimer des contre-revendications. Il était dès lors possible de reprocher aux résidents dont on voulait récupérer la maison de ne pas être en possession des «documents légaux» et de ne pas avoir "versé le loyer dû au käbälé ou à la municipalité 68 ». Cette judiciarisation engendra de nouvelles dynamiques dont la formalisation de la propriété, qui se transforma en une véritable stratégie en vue de s'assurer l'accès aux restitutions. C'est dans ce contexte que certains résidents, qui avaient accédé à la propriété par un processus privé, commencèrent à verser un loyer au nom des occupants antérieurs inscrits au käbälé ou auprès de l'Agence pour le Logement - ce qui n'empêchait pas ces résidents de continuer à se considérer comme propriétaires «légaux» de la

66. Entretien de l'auteur avec un ancien maire de Jigjiga, Jigjiga, 24 avril 2012.

67. Entretien de l'auteur avec le directeur de l'Agence pour le Logement, Jigjiga, 20 août 2013.

68. Fondation du comité de restitution, lettre du 23 septembre 1999. 
maison et de la parcelle. D'autres, qu'ils soient originaires des hautes terres ou somalis, cherchèrent à consolider leur droit de résidence en obtenant un titre de propriété auprès de la municipalité, ce qui devint plus aisé après que le Département technique de la ville eut repris ses activités au tournant des années 2000.

La judiciarisation des restitutions de propriétés fut également visible à travers les stratégies élaborées par les résidents originaires des hautes terres en vue de conserver leur maison ou de prévenir les demandes de restitutions. Ils se mirent pour cela en contact avec l'administration du käbälé, de la municipalité ou de l'Agence pour le Logement et firent valoir que leur droit de résidence était conforme à la loi sur la propriété. D'autres résidents originaires des hautes terres firent valoir auprès de la Cour des certificats de propriété émis par la municipalité sous le därg. Les procédures judiciaires qui suivirent ont montré que le käbälé était chargé de faire la lumière sur le statut locatif des résidences, alors que la municipalité s'occupait d'authentifier la valeur juridique des certificats de propriété qu'elle avait émis sous le régime du därg ${ }^{69}$. Mais dans la majorité des cas, il fut très difficile pour les fonctionnaires de déterminer la base légale sur laquelle évaluer les demandes de restitution ou les contestations des résidents.

Toujours est-il que les chances de voir sa demande de restitution reconnue et exécutée par la Cour dépendaient désormais de plus en plus de preuves concrètes. Dans les faits, cela impliquait de pouvoir accéder aux justificatifs légaux pertinents, «prouvant» qu'une maison avait été occupée et possédée avant la guerre. Cette démarche s'est avérée bien difficile dans la mesure où de nombreux titres et documents avaient disparu avec la destruction des archives ou le départ forcé des habitants durant les différents conflits. Par conséquent, les procédures judiciaires prenaient souvent en considération les témoignages d'anciens voisins pour combler l'absence de preuves formelles de propriété. D’autres requérants décidèrent de consulter les archives de la ville de Harar où des copies de documents officiels avaient été conservées au cours des régimes précédents. Selon les Somali ayant fait cette démarche, l'archiviste était tout à fait disposé à fouiller les registres, attendant une petite rétribution financière en échange du document trouvé. Par ce biais, de nombreux requérants parvinrent à se procurer des reçus prouvant les versements de taxes ainsi que des certificats de propriété émis durant la période impériale. Ces documents, reconnus par le tribunal ou la municipalité, fournirent les preuves nécessaires à leurs demandes antérieures.

69. Cour suprême régionale, lettre enregistrée, 18 janvier 2005. 


\section{LE DOSSIER}

134 Propriété et citoyenneté dans l'Afrique des villes

Cet effort d'«exhumer» des documents et titres de la période impériale est particulièrement intéressant par rapport à la constitution de la propriété en Éthiopie. Les différents gouvernements ont élaboré et modifié les lois relatives à la propriété en fonction des idéologies dominantes, en parties contradictoires. Il en a résulté une certaine sédimentation dans les façons de formuler la loi, ce que reflètent les titres et les documents juridiques. Néanmoins, les institutions gouvernementales ne commencèrent à jouer un rôle notable dans la réglementation de la propriété que sous le därg, la municipalité de Jigjiga ne contrôlant guère que la moitié des parcelles urbaines durant la période impériale ${ }^{70}$. De plus, une part substantielle des propriétés urbaines était alors surtout reconnue et validée à travers des relations sociales qui n'étaient pas matérialisées - ou seulement partiellement - par des documents écrits. Ces couches sédimentaires furent néanmoins fouillées et devinrent des éléments clés de la reconnaissance et de la légitimation des processus de restitutions après la chute du därg. Les reçus des taxes versées durant la période impériale étaient notamment présentés comme des «preuves légales » servant désormais à justifier la propriété sous le nouveau régime. Ainsi, ces documents initialement émis pour un père, voire un grand-père, devaient maintenant valider un droit de propriété. Ce faisant, la validation d'une demande de restitution sur la base de telles «preuves» a contribué à la réinvention d'un régime de propriété antérieur, à la production de l'autorité étatique, et à la remise en œuvre de l'État.

Cette diversion des preuves est capitale au sein d'un ordre normatif pluriel où les frontières catégorielles sont des plus ambiguës. Parker Shipton ${ }^{71} \mathrm{a}$ bien montré l'enjeu que représente le contrôle du «langage» et des «traductions » dans la définition de la légitimité de la preuve. Le cas de Jigjiga est également révélateur des façons dont le pluralisme normatif a promu le «shopping 72 » visant à se procurer les documents grâce auxquels une telle "traduction" pouvait se faire. Récemment, une personne réclamant la restitution de «sa » propriété est ainsi parvenue à se procurer le certificat de propriété de son père (datant de la période impériale) en achetant cette "preuve » à un courtier immobilier. Mais tandis que ce requérant avait pu bénéficier de ses affiliations claniques et s'appuyer sur son réseau, il ne parvint finalement pas à voir sa demande validée par la municipalité ou le tribunal, en raison de son retour tardif à Jigjiga au début des années 2000 et de son incapacité à établir des relations auprès de l'administration locale. Car face aux nouvelles preuves

70. T. Eshete, A History of Jigjiga Town..., op. cit., p. 199.

71. P. Shipton, «Land and Culture in Tropical Africa: Soils, Symbols, and the Metaphysics of the Mundane», Annual Review of Anthropology, vol. 23, 1994, p. 347-377.

72. K. von Benda-Beckmann, «Forum Shopping and Shopping Forums: Dispute Processing in a Minangkabau Villabe in West Sumatra», Journal of Legal Pluralism, n 19, 1981, p. 117-159. 
apportées, la rétention ou la mise à l'écart des titres et documents constituaient une véritable stratégie visant à protéger l'accès à la propriété. Ainsi, un des rares Arabes de retour à Jigjiga ne parvint pas, malgré ses demandes répétées auprès de la municipalité, à récupérer les maisons de son père - dont je découvris le dossier caché dans l'espace privé d'un employé de la ville. Ce dossier contenait en effet une décision rendue par le tribunal le reconnaissant comme propriétaire, mais par la mise à l'écart de son dossier, les maisons pouvaient rester sous l'administration du käbälé. Ces deux cas montrent l'importance des affiliations claniques, des réseaux gouvernementaux et professionnels que ce soit pour l'accès à la propriété ou, au contraire, pour l'ignorance volontaire des preuves écrites. Ainsi, les droits de propriété dans la ville de Jigjiga dépendent moins des cadres légaux que des façons dont ceux-ci sont mobilisés au sein des relations sociales particulières ${ }^{73}$.

Environ deux décennies se sont écoulées depuis la chute du därg, durant lesquelles les modalités d'accès à la propriété et la définition même de cette dernière ont été renégociées et redessinées à travers le processus de restitutions des propriétés, comme le montre le cas de Jigjiga. La propriété tout comme la citoyenneté ethnique, également négociée, y ont été mises en œuvre par des mécanismes à la fois d'accès et d'exclusion. Les citoyens ont eu recours aux institutions gouvernementales en vue d'obtenir la reconnaissance et le règlement de leurs revendications de propriété actuelles ou anciennes. Les représentants du gouvernement ont pour leur part tenté de reconnaître et traiter ces demandes conformément à la loi. Mais en dépit de ces efforts mutuels dans le maintien et l'exercice de l'autorité politique, les restitutions de propriétés demeurent un sujet de contestation où les revendications autour d'un même objet se croisent et où la propriété est toujours contestée.

Quels enseignements pouvons-nous tirer de ces contestations et de ce qu'elles révèlent de la négociation de l'autorité politique à Jigjiga? Notons tout d'abord que la reconnaissance constitutionnelle de l'identité somalie a fourni un ressort essentiel à l'autorité du gouvernement éthiopien parmi les habitants somalis. Mais en reconnaissant la citoyenneté ethnique, le nouveau régime a également rendu possible la réinterprétation des cadres juridiques non seulement des citoyens et de leur quotidien, mais également de la propriété urbaine. La citoyenneté ethnique et le nouvel ordre ethno-fédéral furent ainsi mobilisés par une partie de la communauté somalie autochtone en vue de légitimer leurs demandes de restitutions de propriétés. Ces der-

73. Voir S. A. Bezabeh, «Citizenship and the Logic...», art. cit. 


\section{LE DOSSIER}

136 Propriété et citoyenneté dans l'Afrique des villes

nières contrevenaient cependant à certains droits accordés par les régimes précédents et dont l'application avait été attribuée à des institutions gouvernementales bien précises. Les résidents originaires des hautes terres occupant les maisons et les parcelles réclamées étaient ainsi les premiers touchés par ces développements. En résumé, la reconnaissance des revendications somalies comme "prioritaires » conféra une certaine autorité aux institutions gouvernementales disposant des moyens pour la mettre en œuvre, mais elle affecta cette même autorité du point de vue des citoyens dont les acquis étaient remis en cause par ces réformes.

Cette possible et néanmoins concrète érosion de l'autorité politique dans le processus de restitution incita le gouvernement à intervenir de diverses façons en vue de maintenir une cohérence légale et une certaine conformité à la loi. Cependant, les contradictions inhérentes au fait de prioriser les demandes de restitution comme «des revendications antérieures relative à un droit de propriété à l'encontre de demandes actuelles concurrentes ${ }^{74}$ » n'ont pas été résolues. C'est dans ce contexte que les acteurs à l'intérieur et à l'extérieur de l'appareil bureaucratique ont négocié les aspects juridiques de la citoyenneté et les catégories de la propriété, (re)produisant ainsi une pluralité d'ordres normatifs déterminant les règles des restitutions. Pour des citoyens de diverses communautés ethniques, ce «pluralisme post-conflit ${ }^{75}$ » représentait non seulement l'opportunité d'aller faire son «marché » dans les interstices entre les différente institutions impliquées dans la régulation de la propriété ou à l'intérieur de chacune d'entre elles, mais également la possibilité de justifier leurs demandes par le droit. Les institutions gouvernementales se transformèrent alors en plateformes où les parties en conflit devaient s'attirer les faveurs du fonctionnaire en charge du dossier - en revendiquant leur droit et en fournissant des preuves. Comme nous avons pu le voir, les catégories liées à la citoyenneté ethnique et la propriété représentent des mécanismes potentiellement antagonistes quant aux modalités d'accès au foncier, même si elles dérivent du même droit écrit. Un tel paradoxe a incité les requérants, de même que ceux qui s'y opposaient, à manipuler les catégories de citoyenneté légale et de propriété, quelle que soit l'issue du jugement. Ainsi, l'État a marqué sa présence, certes incohérente, dans l'ordre discursif, et il a acquis «son autorité à autoriser ${ }^{76}$ ». L'État s'est par conséquent vu attribué un rôle de plus en plus considérable dans le contrôle du foncier dans les basses terres orientales de l’Éthiopie.

74. M. Blacksell et K. M. Born, «Private Property Restitution...», art. cit., p. 188.

75. J. Unruh, «Land Tenure and Legal Pluralism in the Peace Process », Peace and Change, vol. 28, $n^{\circ} 3,2003$, p. 352-377.

76. T. B. Hansen et F. Stepputat (dir.), States of Imagination..., op. cit., p. 8. 
Les processus de restitutions de propriétés à Jigjiga représentent un moment intéressant de négociation de l'autorité politique et de production de l'État, durant lequel divers acteurs ont eu recours à des catégories légales en mettant en avant des documents légaux, réactivant ainsi des liens antérieurs avec les institutions gouvernementales. Les modalités d'accès, les revendications et les exclusions ont ainsi été déterminées et consolidées à travers des discours et des pratiques façonnant et reconfigurant la propriété ${ }^{77}$. C'est à travers la soumission - et la rétention - de documents légaux que des demandes relatives aux propriétés sont devenues lisibles ou illisibles, que l'autorité politique s'est vue renforcée ou affaiblie, et qu'ont été tracés les contours de la frontière séparant l'État et la société ${ }^{78}$. L'État est donc mis en œuvre à travers des pratiques sociales quotidiennes et variées, mais aussi à travers la construction, la reconstruction ou la déconstruction de la propriété.

Rony Emmenegger

Université de Zurich

Traduction: Jean-Nicolas Bach

Abstract

Between Power and Authority: Enacting the State through Property in Urban Jigiiga, Ethiopia

This paper looks at urban property restitution as a juncture where political authority is negotiated and where the state is enacted. Based on ethnographic fieldwork in the city of Jigiiga, Ethiopia, it discusses the role of legal property and ethnic citizenship categories in the negotiation of access and property in a post-conflict context. Thereby, it illustrates how legal categories have been entangled in plural normative orders regulating cases of property restitution and setting the conditions for the (re)making of property relations. This inquiry also reveals that legal property and ethnic citizenship categories can constitute contradicting mechanisms of access and exclusion, despite being codified within the same body of statutory law. Overall, this paper draws attention to the inherent ambiguities of state formation processes and the complex dynamics at the margins of the state.

77. N. L. Peluso et C. Lund, «New Frontiers...», art. cit., p. 668. Voir également N. Blomley, «Cuts, Flows, and the Geographies of Property», Law, Culture and the Humanities, vol. 7, n² 2, 2010, p. 205. 78. Voir V. Das et D. Poole (dir.), Anthropology..., op. cit., p. 10; T. Mitchell, «The Limits of the State...», art. cit. 